\title{
Primary adrenal lymphoma: three case reports and review of Japanese cases
}

\author{
Shigeaki Nakazawa $\cdot$ Motohide Uemura \\ Takeshi Ujike · Kazutoshi Fujita · Tetsuya Takao • \\ Yasushi Miyagawa $\cdot$ Norio Nonomura
}

Received: 27 August 2014/Accepted: 27 October 2014/Published online: 4 November 2014

(C) The Japan Society of Clinical Oncology 2014

\begin{abstract}
Although primary adrenal lymphoma (PAL) is thought to be extremely rare, its prognosis is much worse than that of other extranodal malignant lymphomas. There are so far about 130 reported cases in the English literature, while 186 cases have been reported in the Japanese literature. Thus, the incidence of PAL in the Japanese population may be higher than in other races. The typical characteristics of PAL in Japanese patients are similar to those previously reported in Western literature: it predominantly occurs in male and elderly patients and involves bilateral involvement of the adrenal glands, which frequently leads to adrenal insufficiency. Here, we review three recent cases of PAL at our hospital, and analyze data from our institution regarding patients with PAL from 2002 to 2014. On biochemical analysis, median levels of sIL2R (5027.5 U/mL) and LDH (1111.46 U/L) were elevated in Japanese PAL patients compared to other adrenal tumors. It is critical that clinicians be familiar with the traits of PAL, especially for its differential diagnosis from adrenal large tumors.
\end{abstract}

Keywords Primary adrenal lymphoma $\cdot$ Lactate dehydrogenase $\cdot$ Soluble interleukin-2 receptor

\section{Introduction}

While lymphoma can occur in any body organ, primary adrenal lymphoma (PAL) is extremely rare, with only

S. Nakazawa · M. Uemura ( $₫) \cdot$ T. Ujike $\cdot$ K. Fujita ·

T. Takao $\cdot$ Y. Miyagawa $\cdot$ N. Nonomura

Department of Urology, Osaka University Graduate School of

Medicine, 2-2 Yamadaoka, Suita City, Osaka 565-0871, Japan

e-mail: uemura@uro.med.osaka-u.ac.jp about 130 cases reported in the English literature. PAL is highly aggressive and results in death within weeks if left untreated. As such, preoperative differentiation between PAL and other potentially malignant tumors is imperative for proper treatment [1]. However, correctly diagnosing PAL by radiological methods remains a key challenge in treatment of this disease.

Lactate dehydrogenase (LDH) and soluble interleukin-2 receptor (sIL2R) are considered potential prognostic factors for lymphoma and may predict the clinical course of the disease [2]. However, few reports are available on whether LDH and sIL2R effectively distinguish PAL from other adrenal tumors.

Herein, we report our three cases of PAL and review the reported 186 cases in Japan. The objective of this paper is to detail the characteristics of PAL in Japan, and to examine whether biomarkers such as LDH and sIL2R are useful in its differential diagnosis.

\section{Case report}

In case 1, a 62-year-old man had visited the hospital because of right quadrant pain in December 2002. His past medical history was hepatitis B infection and tuberculosis. Contrast-enhanced computed tomography (CT) revealed a $3.9 \mathrm{~cm}$ right adrenal mass that looked homogenous and not enhanced. Because the patient showed no hormonal dysfunction upon endocrinological examination, he was followed up as an incidental non-functioning adenoma. 3 months later, his body weight was reduced by $8 \mathrm{~kg}$, and CT detected rapid cancer growth, to a size of $6 \mathrm{~cm}$, and invasion of the liver (Fig. 1a). Laboratory investigations showed increased LDH to $397 \mathrm{U} / \mathrm{mL}$. Under the diagnosis of right adrenal adrenocortical carcinoma, we performed open right adrenalectomy. Because the tumor had firm 
Fig. 1 CT scans demonstrated predominant enlargement of adrenal gland. In cases 2 and 3 , CT scans also revealed kidney masses. a Case 1 , b case 2 and c case 3
Fig. 2 Positron emission tomography (PET) revealed uptake in the adrenal gland and kidney mass. a Case 2 and b case 3
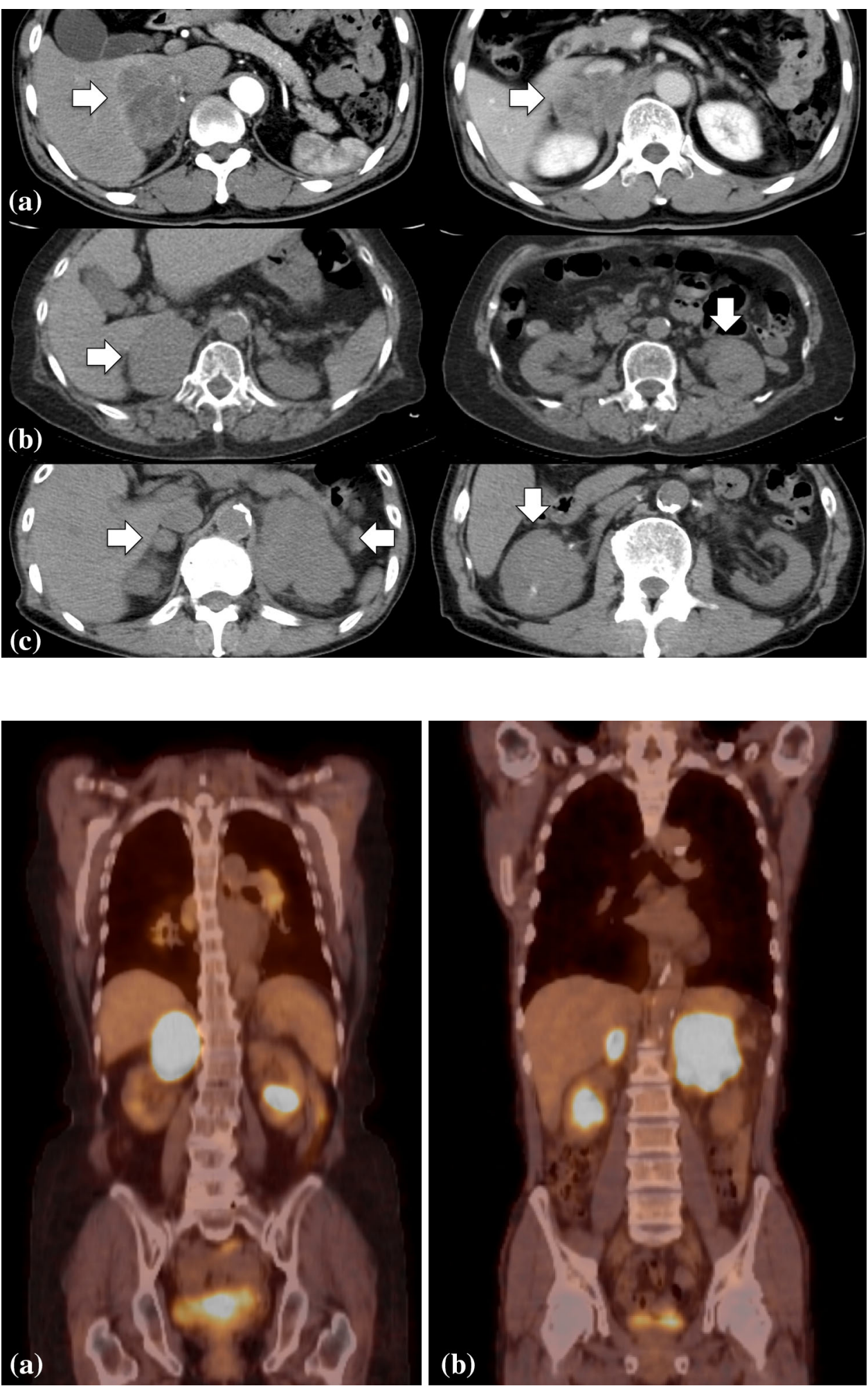

adhesion to the right kidney and liver, we had to resect the right kidney, the posterior segment of the liver and an adjacent portion of the inferior vena cava. Operation time was $425 \mathrm{~min}$, and blood loss was $3480 \mathrm{~mL}$. Histopathological examination revealed diffuse large B cell lymphoma
(DLBCL). Adjuvant chemotherapy according to the CHOP-regimen (cyclophosphamide, doxorubicin, vincristine, and prednisone) was started. After the four cycles of chemotherapy, he is still alive with no evidence of disease 9 years after diagnosis. 
Fig. 3 Resected specimen in case 2. a Adrenal tumor and b left kidney
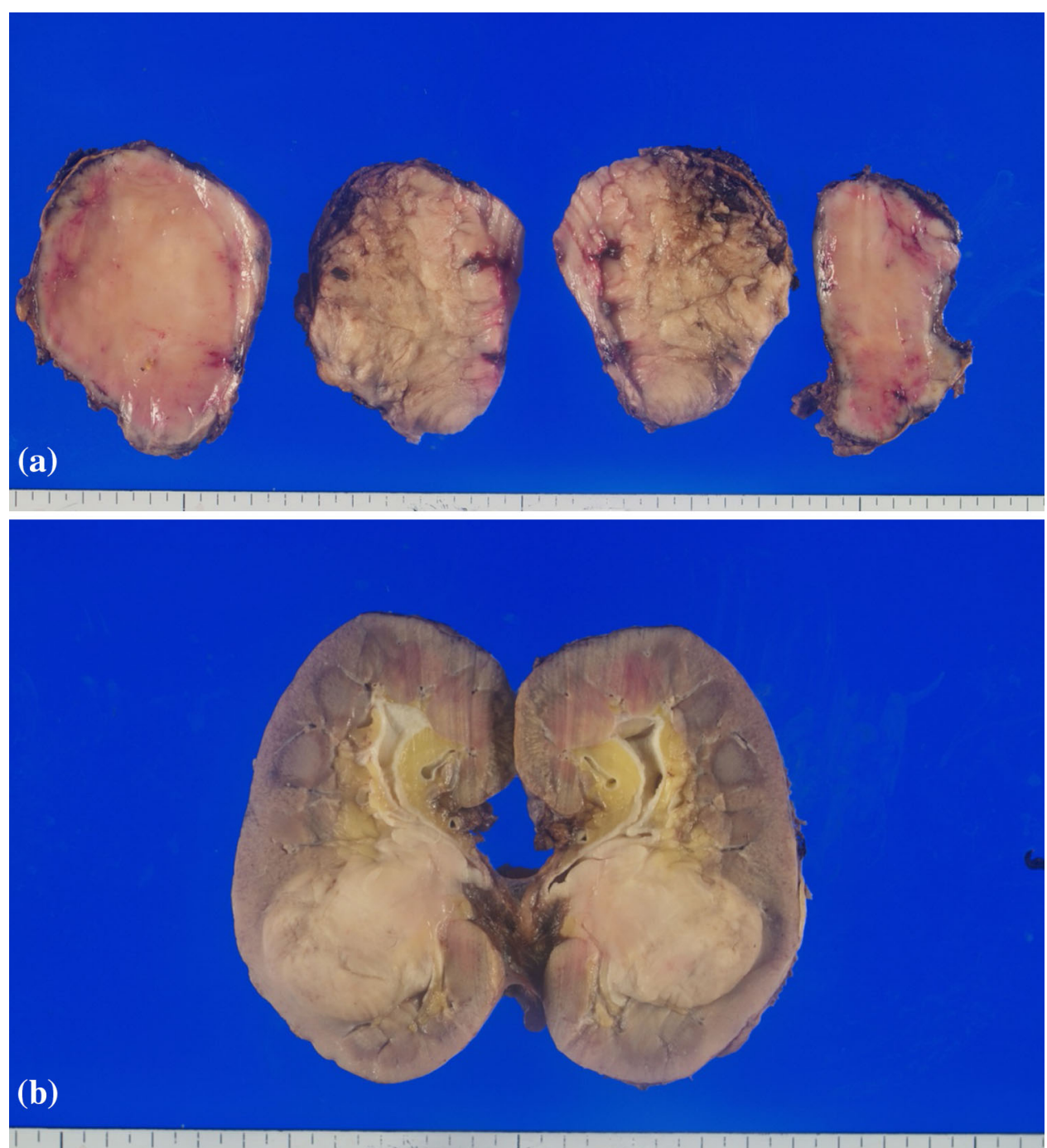

In case 2, a 77-year-old woman had been undergoing CT scans regularly to check for recurrence of sigmoid colon cancer. In September 2007, CT scans revealed a right $3.5 \mathrm{~cm}$ adrenal mass. Endocrinological tests revealed no hormonal dysfunction. After 2 months, F-18 fluorodeoxyglucose positron emission tomography (FDG-PET) showed increased uptake at the right adrenal gland, which measured $5.5 \mathrm{~cm}$, and also at the left kidney, which was not detected by the previous CT (Figs. 1b, 2a). Laboratory investigations showed elevated levels of LDH $(532 \mathrm{U} / \mathrm{mL})$. In December 2007, under the diagnosis of right adrenal adrenocortical carcinoma with metastasis to the left kidney, we performed concomitant resection of the right adrenal mass and the left kidney (Fig. 3). In this case, the tumor had no adhesion with adjacent organs. Operation time was $355 \mathrm{~min}$, and blood loss was $1550 \mathrm{~mL}$. The histopathological diagnosis was DLBCL. Although the patient received adjuvant CHOP chemotherapy, she died from progressive disease 18 months after surgery.
In case 3, a 66-year-old man was referred to our hospital for further examination of a right kidney mass and left adrenal mass lesions in June 2010. He complained of general fatigue, appetite loss, and weight loss by $7 \mathrm{~kg}$ in a month. His past history was unremarkable except for hypertension and chronic renal failure. CT scans indicated enlargement of the bilateral adrenal glands and right kidney mass (Fig. 1c). Biochemical analysis revealed elevated levels of LDH (491 U/L) and sIL2R (8804 U/mL). FDGPET demonstrated that there was strong uptake in the bilateral adrenal glands, right kidney mass, and paraaortic lymph nodes (Fig. 2b). Based on the laboratory data and imaging analysis, malignant lymphoma was highly suspected at this point. To make a definitive diagnosis, open biopsy of the left adrenal mass was performed. The histopathological diagnosis was DLBCL (Fig. 4). During CHOP chemotherapy, he died of subarachnoid hemorrhage 21 months after diagnosis. Table 1 shows the characteristics of our patients. 


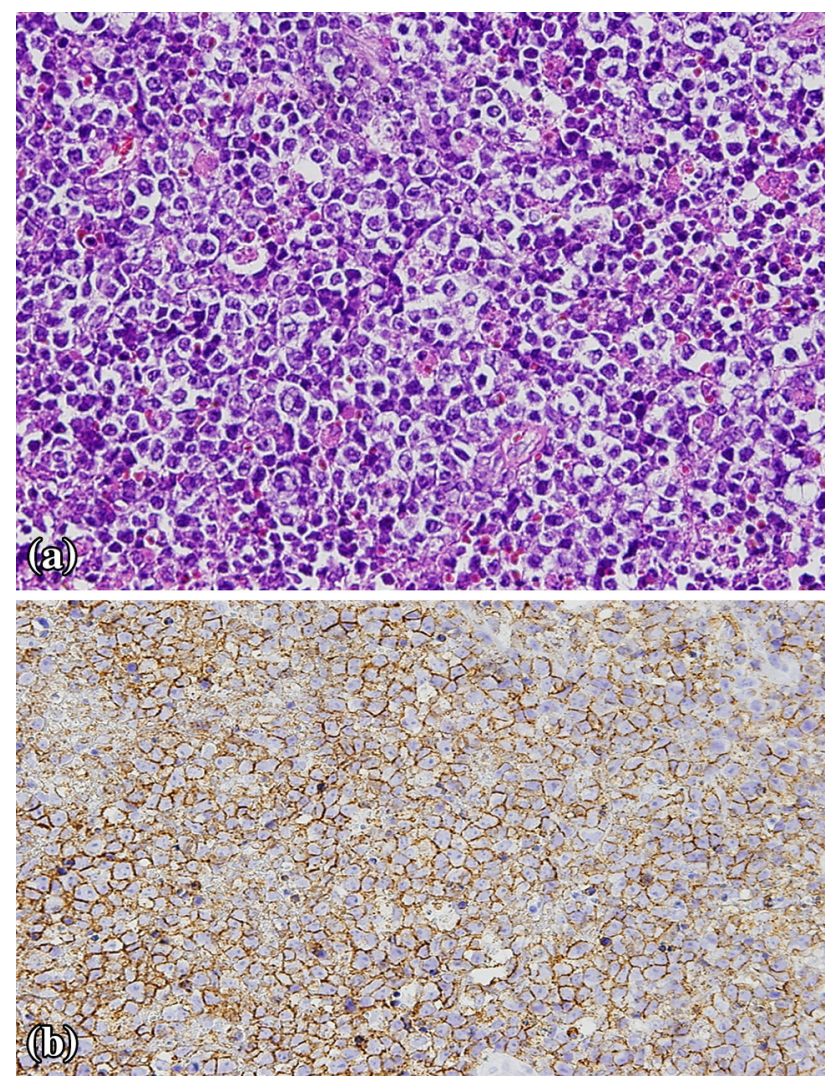

Fig. 4 a Histopathological examination showing diffuse large cell infiltration (H\&E stain). b Immunohistochemical staining demonstrating that tumor cells were positive for CD20

\section{Discussion}

PAL is an extremely rare entity, accounting for less than $1 \%$ of all non-Hodgkin's lymphomas (NHL) and only $3 \%$ of primary extranodal lymphomas [3, 4].

There are so far about 130 reported cases in the English literature, while 186 cases have been reported in the Japanese literature. We reviewed the reported cases in Japan from 1982 to 2012 in Table 2. PAL occurs with a male:female ratio of 2.2:1 and median age is 67.1 years (range 17-87). Up to $90.3 \%$ of patients with PAL present with systemic complaints of fever, weight loss, abdominal pain, and appetite loss; $73.1 \%$ of PAL cases showed bilateral adrenal involvement with a median maximum diameter of $7.12 \mathrm{~cm}$ at the time of diagnosis. In $38.1 \%$ of PAL cases, an adrenal insufficiency was observed even when the neoplasm was small. Based on histopathological and immunophenotyping examinations, about $79.5 \%$ of PAL cases are B cell lymphomas. The most common histological subtype is DLBCL, showing positive expressions of leukocyte antigen CD45 and of B cell marker CD20. Overall survival (OS) rates at 1 and 2 years are 38.2 and $33.9 \%$, respectively, and median OS was 9 months (95\% CI; 7-12 months) (Fig. 5). Kim et al. [5] and Kumar et al. [6] reported that many PAL patients die due to tumor recurrence or severe infections within 1 year. They reported that the mean duration of survival in patients who respond to chemotherapy was $34 \pm 32$ months, whereas
Table 1 Patient characteristics in our institution

$D L B C L$ diffuse large cell lymphoma

* Postoperative analysis

\begin{tabular}{|c|c|c|c|}
\hline & Patient 1 & Patient 2 & Patient 3 \\
\hline Age/sex & 62-year-old male & 77-year-old female & 66-year-old male \\
\hline Chief complaint & Abdominal pain & None & Weight loss \\
\hline Past medical history & $\mathrm{HBV}$ and tuberculosis infection & Sigmoid colon cancer & None \\
\hline $\begin{array}{l}\text { Primary site of } \\
\text { adrenal gland }\end{array}$ & Right & Right & Bilateral \\
\hline Tumor size & $6 \mathrm{~cm}$ & $5.5 \mathrm{~cm}$ & $\begin{array}{l}2.0 \mathrm{~cm} \text { (right) } \\
8.9 \mathrm{~cm} \text { (left) }\end{array}$ \\
\hline $\begin{array}{c}\text { Other site of } \\
\text { lymphoma }\end{array}$ & $\begin{array}{l}\text { Direct invasion to liver and } \\
\text { inferior vena cava }\end{array}$ & Left kidney & Right kidney \\
\hline sIL2R (U/mL) & $1488^{*}$ & $1150^{*}$ & 8804 \\
\hline LDH (U/L) & 397 & 532 & 499 \\
\hline 18FDG-PET & Not tested & Increased uptake & Increased uptake \\
\hline Treatment & $\begin{array}{l}\text { Surgical resection and } \\
\text { chemotherapy }\end{array}$ & $\begin{array}{l}\text { Surgical resection and } \\
\text { chemotherapy }\end{array}$ & $\begin{array}{l}\text { Open biopsy and } \\
\text { chemotherapy }\end{array}$ \\
\hline Histopathology & DLBCL & DLBCL & DLBCL \\
\hline \multirow[t]{2}{*}{ Prognosis } & 9 years & 18 months & 21 months \\
\hline & $\begin{array}{l}\text { Alive with no evidence of } \\
\text { disease }\end{array}$ & Died of disease & Died of other disease \\
\hline
\end{tabular}


Table 2 Clinical features of primary adrenal lymphoma in the Japanese literature

\begin{tabular}{ll}
\hline Total cases & 186 \\
Age & $67.1 \pm 11.8$ years \\
& $(17-87$ years $)$ \\
Sex (male/female) & $128 / 58(2.2: 1)$ \\
Associated symptoms & $168(90.3 \%)$ \\
Primary site of adrenal gland (bilateral/ & $136(73.1 \%) / 52(27.9 \%)$ \\
$\quad$ unilateral) & \\
Tumor size & $7.12 \pm 2.99 \mathrm{~cm}$ \\
Adrenal insufficiency & $71(38.1 \%)$ \\
Central nerve invasion & $13(6.9 \%)$ \\
Histopathology & $101(54.3 \%) / 85(45.7 \%)$ \\
DLBCL/others & $148(79.5 \%) / 38(20.5 \%)$ \\
B cell type/others & \\
Biochemical marker & $656(173-3899)$ \\
LDH (U/L) & $4524(555-14500)$ \\
sIL2R (U/mL) & \\
Definitive diagnostic procedure & $74(39.7 \%)$ \\
Needle biopsy (US or CT guided) & $27(14.5 \%)$ \\
Open biopsy & $41(22.0 \%)$ \\
Tumor resection & $21(11.2 \%)$ \\
Autopsy & $23(12.3 \%)$ \\
Others &
\end{tabular}

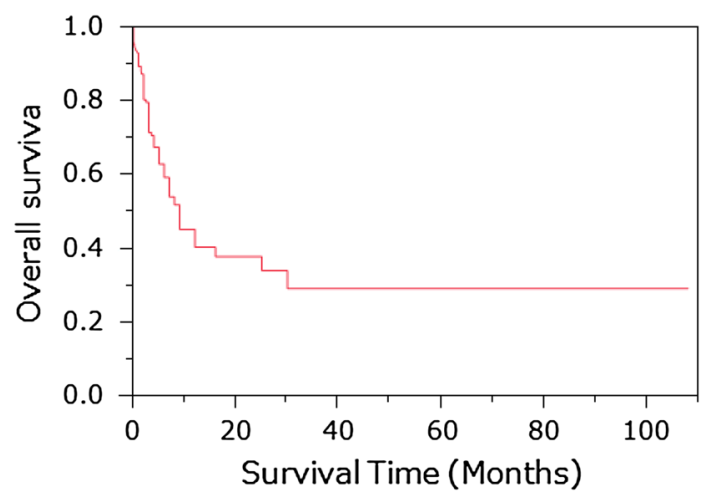

Fig. 5 Overall survival of 186 patients with primary adrenal lymphoma in Japan (overall survival was calculated from the date of diagnosis until death from any cause or until the last follow-up. Survival curves were estimated by the Kaplan-Meier method)

the mean duration of survival in patients without response for chemotherapy was only $3.6 \pm 3.9$ months.

The adrenal gland is a common site for neoplastic disease, and every adrenal tumor has to be diagnosed properly with regard to its malignancy potential and hormonal activity. Malignant adrenal tumors include adrenocortical carcinoma (ACC), PAL and adrenal metastasis from other organs. Unlike treatment for other adrenal malignant tumors, the therapeutic strategy against PAL is systemic chemotherapy, and so it is critical to assign the correct
Table 3 Comparison of preoperative levels of $\mathrm{LDH}$ and sIL2R between PAL and other adrenal tumors

\begin{tabular}{cccc}
\hline & PAL & Other adrenal tumors & $p$ value \\
\hline LDH & $1111.4 \pm 98.0(95 \%$ & $215.9 \pm 88.3(95 \%$ & $<0.0001$ \\
(U/L) & CI; 822.2-1400.7) & CI; $197.9-233.9)$ & \\
sIL2R & $5027.5 \pm 555.8(95 \%$ & $525.7 \pm 1283.6$ & 0.0018 \\
(U/ & CI; 3827.5-6227.6) & $(95 \% \mathrm{CI} ;$ & \\
$\mathrm{mL})$ & & $356.2-695.3)$ & \\
\hline
\end{tabular}

differential diagnosis at an early stage. Accurate diagnosis of PAL by CT is complicated by its similarity in appearance with ACC: it tends to appear as complex masses with variable density, demonstrating no distinctive pathognomonic features [1, 7, 8]. And while positron emission tomography (PET) using 18 fluorodeoxyglucose can distinguish between malignant and benign adrenal lesions with $95 \%$ accuracy, it cannot distinguish PAL from ACC or other metastatic diseases, as all these show increased uptake of 18 FDG $[1,7,8]$. Thus, radiological differential diagnosis between PAL and ACC remains difficult, to the extent that correct diagnoses of PAL are often not made until during surgery $(22.0 \%$ of cases $)$ or postmortem autopsy (11.2\% of cases), as shown in Table 1.

The level of serum LDH and sIL2R closely tracks with the activity of non-Hodgkin's lymphoma, and these biomarkers are known to be prognostic parameters in patients with malignant lymphoma $[9,10]$. However, there are few reports about these levels in patients with PAL. We experienced 160 cases of surgical resection or biopsy of adrenal tumors from May 2002 to April 2012 at our institution, 3 cases of which were PAL. We compared the level of LDH and sIL2R of PAL to other adrenal tumors. LDH and sIL2R of other adrenal tumors are collected from medical records at our institution. Statistical assessments were carried out using the Mann-Whitney $U$ test, and $p<0.05$ was considered statistically significant.

The LDH data were collected from 125 cases of PAL and 154 cases of other adrenal tumors. The level of LDH in PAL was $1111.4 \pm 98.0 \mathrm{U} / \mathrm{L}(95 \% \mathrm{CI} ; 822.2-1400.7$ $\mathrm{U} / \mathrm{L})$, compared to $215.9 \pm 88.3 \mathrm{U} / \mathrm{L} \quad(95 \% \quad \mathrm{CI}$; 197.9-233.9 U/L) for other tumors. The comparison of LDH between both groups showed a significant difference $(p<0.001)$. The data for sIL2R were collected in 80 cases of PAL and 15 cases of other adrenal tumors, with the level of sIL2R for the two groups being $5027.5 \pm 555.8 \mathrm{U} / \mathrm{mL}$ $(95 \% \mathrm{CI} ; 3827.5-6227.6 \mathrm{U} / \mathrm{mL})$ and $525.7 \pm 1283.6$ (95\% CI; 356.2-695.3 U/mL), respectively. This difference was also statistically significant $(p=0.0018)$ (Table 3). Our study suggests that measurement of LDH and sIL2R may be useful in diagnosing suspected malignant lymphoma in patients with adrenal enlargement as in our case 3 . 
Conflict of interest The authors declare that they have no conflict of interest.

\section{References}

1. Ozimek A, Diebold J, Linke R et al (2008) Bilateral primary adrenal non-Hodgkin's lymphoma and primary adrenocortical carcinoma-review of the literature preoperative differentiation of adrenal tumors. Endocr J 55(4):625-638

2. Goto N, Tsurumi H, Goto H et al (2012) Serum soluble interleukin-2 receptor (sIL-2R) level is associated with the outcome of patients with diffuse large $B$ cell lymphoma treated with R-CHOP regimens. Ann Hematol 91(5):705-714

3. Dunnick NR, Korobkin M (2002) Imaging of adrenal incidentalomas: current status. AJR Am J Roentgenol 179(3):559-568

4. Anderson T, DeVita VT Jr, Simon RM et al (1982) Malignant lymphoma. II Prognostic factors and response to treatment of 473 patients at the National Cancer Institute. Cancer 50(12): $2708-2721$
5. Kim YR, Kim JS, Min YH et al (2012) Prognostic factors in primary diffuse large B-cell lymphoma of adrenal gland treated with rituximab-CHOP chemotherapy from the Consortium for Improving Survival of Lymphoma (CISL). J Hematol Oncol 5:49

6. Kumar R, Xiu Y, Mavi A et al (2005) FDG-PET imaging in primary bilateral adrenal lymphoma: a case report and review of the literature. Clin Nucl Med 30(4):222-230

7. Low G, Dhliwayo H, Lomas DJ (2012) Adrenal neoplasms. Clin Radiol 67(10):988-1000

8. Wang J, Sun NC, Renslo R, Chuang CC et al (1998) Clinically silent primary adrenal lymphoma: a case report and review of the literature. Am J Hematol 58(2):130-136

9. Kamihira S, Atogami S, Sohda H et al (1994) Significance of soluble interleukin-2 receptor levels for evaluation of the progression of adult T-cell leukemia. Cancer 73(11):2753-2758

10. Motokura T, Kobayashi Y, Fujita A et al (1995) Clinical significance of serial measurement of the serum levels of soluble interleukin-2 receptor and soluble CD8 in malignant lymphoma. Leuk Lymphoma 16(3-4):355-362 